\title{
An Evaluation Method for Harmonic Emission Level Based on Principal Component Regression
}

\author{
Liang Wang ${ }^{1}$, Shugang Wang ${ }^{1}$, Dongliang $\mathrm{Wu}^{1}$, Haihua $\mathrm{Liu}^{2}$, Jie Wang ${ }^{3 *}$ \\ ${ }^{1}$ State Grid Yangzhou Power Supply Company, Yangzhou 225001, China \\ ${ }^{2}$ State Grid Nantong Power Supply Company, Nantong 226006, China \\ ${ }^{3}$ Ningneng Electric Design Limited Company, Nanjing 210000, China
}

Corresponding Author Email: xiads@nrec.com

https://doi.org/10.18280/ejee.210503

Received: 17 April 2019

Accepted: 30 July 2019

\section{Keywords:}

harmonic emission level, evaluation, principal component regression (PCR),

power system

\begin{abstract}
To identify the responsible party of harmonic pollution, this paper puts forward a novel estimation method for harmonic emission level based on principal component regression (PCR). After introducing the principles of the PCR, the author set up a regression equation based on the complex relationship between harmonic voltage, harmonic current and harmonic impedance of the supply system at the point of common coupling (PCC). Then, the PCR was introduced to estimate the harmonic emission levels of the supply system and the consumer at the PCC. The validity of our method was verified through both simulation and field test. The results show that our method can accurately estimate the harmonic emission level, while overcoming the multicollinearity of independent variables and retaining important information in regression.
\end{abstract}

\section{INTRODUCTION}

With the growing application of nonlinear loads and distributed generators, the power system is faced with an increasingly severe harmonic pollution [1-2]. To solve the problem, it is imperative to clarify the responsibilities for the pollution and differentiate the harmonic emission levels between the supply system and the consumer [3-6].

In relevant studies, the harmonic emission level is mainly evaluated based on the harmonic contributions of the supply system and the consumer at the point of common coupling (PCC) [7-10]. Many evaluation methods have emerged, including Kalman filter [11-12], harmonic state estimation [13], k-nearest neighbors (kNN) algorithm [14], empirical mode decomposition [15] and distributed measurements [6]. In practice, however, the harmonic emission level is generally evaluated by statistical methods [16].

The fluctuation method and its variants are the earliest statistical evaluation approaches for harmonic emission level [17-18]. By this method, the harmonic impedance of the power system is calculated as the ratio of voltage and current measured at the PCC, and used to identify the responsibilities for harmonic pollution between the power system and the consumer. Despite its simplicity, the fluctuation method may be inaccurate if the voltage and current are not measured accurately.

Later, Zhang and Yang [19] proposed the binary linear regression method, which estimates the resistance component of the harmonic impedance. However, this method performs poorly if the measured voltage and current at the PCC contain singular values. Che and Yang [20] reduced the influence of the singular values through weighting by the robust regression method, but this strategy overlooks the correlation between independent variables in statistical analysis. Then, Huang and
$\mathrm{Xu}$ [21] introduced the partial least squares (PLS) regression to evaluate harmonic emission level. Compared to the robust regression method, the LS regression identifies information and noise in the power system accurately, and determines the correlation between variables based on statistical features.

The above methods improve the estimation accuracy of harmonic emission level to different degrees. Nevertheless, there are two common problems with these strategies. First, there are too many independent variables in these methods, increasing the modelling complexity. Second, some important data may get lost due to the correlation between independent variables. Because of the two problems, the existing estimation methods for harmonic emission level may be inefficient in extracting the information from dependent variables that has powerful explanatory ability. In severe cases, this type of information is entirely lost.

This paper proposes a method to estimate harmonic emission level based on principal component regression (PCR). In this method, the principal components are selected according to the amount of the information contained, thus overcoming multicollinearity of the independent variables. Moreover, this method can retain as much information of important independent variables as possible. In this way, the harmonic emission level of both supply system and the consumer can be estimated at a high accuracy.

\section{THE PCR}

The PRC is a statistical method that expresses the information as the linear combination of the least variables [22]. Let by $\left\{x_{1}, x_{2}, \ldots, x_{p}\right\}$ be $p$ independent variables in the problem. Then, the dataset of $n$ samples can be described as: 


$$
X=\left(\begin{array}{cccc}
x_{11} & x_{12} & \cdots & x_{1 p} \\
x_{21} & x_{22} & \cdots & x_{2 p} \\
\vdots & \vdots & \vdots & \vdots \\
x_{n 1} & x_{n 2} & \cdots & x_{n p}
\end{array}\right)=\left(X_{1}, X_{2}, \cdots, X_{p}\right)
$$

The PCR is a linear transform between the $p$ independent variables:

$$
\left\{\begin{array}{c}
F_{1}=a_{11} X_{1}+a_{12} X_{2}+\cdots+a_{1 p} X_{p} \\
F_{2}=a_{21} X_{1}+a_{22} X_{2}+\cdots+a_{2 p} X_{p} \\
\cdots \\
F_{p}=a_{p 1} X_{1}+a_{p 2} X_{2}+\cdots+a_{p p} X_{p}
\end{array}\right.
$$

where, $a_{-} i j$ is the principal component coefficient. Equation (2) can be rewritten as

$$
F=A X
$$

where, $F=\left(F_{1}, F_{2}, \ldots, F_{p}\right)^{T} ; X=\left(X_{1}, X_{2}, \ldots, X_{p}\right)^{T} ; A$ is the score matrix.

Let $\lambda_{1}, \lambda_{2}, \ldots, \lambda_{P}$ be the $p$ eigenvalues of the score matrix $A$ that satisfy $\lambda_{1} \geq \lambda_{2} \ldots \geq \lambda_{n}$, and $a_{j}$ be the corresponding eigenvectors. Then, the variance of $F_{1}$ can be expressed as

$$
\operatorname{Var}\left(F_{1}\right)=a_{1} X X^{T} a_{1}^{T}=\lambda_{1}
$$

Similarly, it can be derived that $\operatorname{Var}\left(F_{i}\right)=\lambda_{i}$. Thus, the variances of principal components decrease in turn. Besides, the covariances of the principal components can be computed by:

$$
\begin{aligned}
& \operatorname{Cov}\left(a_{i}^{T} X^{T}, a_{j} X\right)=a_{i}^{T}\left(\sum_{k=1}^{p} \lambda_{k} a_{k} a_{i}^{T}\right) a_{j} \quad(i \neq j) \\
& =\sum_{k=1}^{p} \lambda_{k}\left(a_{i}^{T} a_{k}\right)\left(a_{k}^{T} a_{j}\right)=0
\end{aligned}
$$

Through the above transform, the independent variables $\left\{x_{1}\right.$, $\left.x_{2}, \ldots, x_{p}\right\}$ become a new set of variables $\left\{F_{1}, F_{2}, \ldots, F_{p}\right\}$, whose variances decrease in turn.

Let $F_{1}$ be the first principal component to replace the original $p$ variables. This component must contain as much information of the $p$ variables as possible. In other words, the variance $\operatorname{Var}\left(F_{l}\right)$ should be maximized. If $F_{l}$ cannot cover all the information of the $p$ variables, the second principal component $F_{2}$ should be introduced. Note that the information already covered by $F_{1}$ should not be included in $F_{2}$, because $\operatorname{cov}\left(F_{1}, F_{2}\right)=0$. The third, the fourth..., the $p$ th principal components can be set up by analogy. According to Equation (5), these principal components are not correlated with each other, and their variances decrease in turn $\operatorname{Var}\left(F_{1}\right)>\operatorname{Var}\left(F_{2}\right), \ldots,>\operatorname{Var}\left(F_{p}\right)$. With the decrease of variance, the amount of information contained in each principal component also declines.

In practice, not all the $p$ principal components are identified. Instead, the first $q$ principal components are selected based on their cumulative contribution rate $\mu$, i.e. the variance ratio of a principal component to the total variance of all principal components:

$$
\mu=\frac{\lambda_{i}}{\sum_{i=1}^{p} \lambda_{i}}
$$

Studies have shown that the integrated variable can cover most information of the original variables, if its cumulative contribution rate surpasses $85 \%$ [23]. The important information of the original independent variables can be preserved by selecting the principal components based on the contribution rate. In addition, if the $F_{i}$ value is close to zero, the corresponding independent variables show an approximate linear correlation. After neglecting the relatively small principal components, it is possible to overcome the collinearity between independent variables.

Once the expression of principal components and subvariables are obtained by the PCR, the dependent variables can be regressed to sub-variables of each principal component. Thus, the regression model can be obtained between dependent variables and sub-variables of each principal component. The expression of principal components facilitates the regression modeling between the standardized independent variables and the dependent variables. Finally, the standardized independent variables can be transformed to the original independent variables, completing the regression modeling between original independent variables and dependent variables.

\section{PCR-BASED ESTIMATION OF HARMONIC EMISSION LEVEL}

The equivalent circuit of the supply system and the consumer at the PCC is shown in Figure 1, where $U_{s h}$ is the harmonic voltage source of the supply system, $I_{c h}$ is the equivalent harmonic current source of the consumer, $Z_{s h}$ and $Z_{c h}$ are the harmonic impedances of the supply system and the consumer, respectively, and $I_{p c c h}$ and $U_{p c c h}$ are the harmonic current and harmonic voltage measured at the PCC, respectively. According to the circuit principle, the following equation can be derived:

$$
\dot{U}_{p c c h}=\dot{U}_{s h}-\dot{I}_{p c c h} \dot{Z}_{s h}
$$

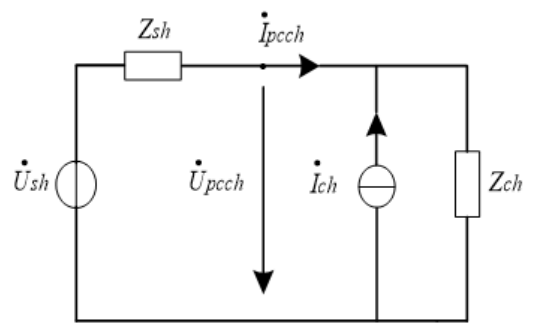

Figure 1. The equivalent circuit of the supply system and the consumer

The real part and the imaginary part can be expressed as multiple linear regression equations, respectively:

$$
\begin{gathered}
U_{p c c h x}=U_{s h x}-I_{p c c h x} Z_{s h x}+I_{p c c h y} Z_{s h y} \\
U_{p c c h y}=U_{s h y}-I_{p c c h x} Z_{s h y}-I_{p c c h y} Z_{s h x}
\end{gathered}
$$


where, $I_{p c c h x}$ and $I_{p c c h y}$ are the real part and the imaginary part of the harmonic current $I_{p c c h}$ measured at the PCC, respectively; $U_{p c c h x}$ and $U_{p c c h y}$ are the real part and the imaginary part of the harmonic voltage $U_{p c c h}$ measured at the PCC, respectively. In multiple linear regression, $I_{p c c h x}$ and $I_{p c c h y}$ are independent variables, while $U_{p c c h x}$ and $U_{p c c h y}$ are dependent variables. The regression coefficients $Z_{s h x}, Z_{s h y}, U_{s h x}$ and $U_{s h y}$ can be obtained by solving Equations (8) and (9) by the PCR.

To reduce the background harmonic fluctuation, the data used to calculate $Z_{s h}$ and $U_{s h}$ are often divided into $M$ sections. Then, the statistical values of $Z_{s h}$ and $U_{s h}$ can be respectively obtained by:

$$
\begin{gathered}
\bar{Z}_{s h}=\frac{1}{M}\left(\sum_{m=1}^{M} Z_{s h x m}+j \sum_{m=1}^{M} Z_{s h y m}\right) \\
\bar{U}_{s h}=\sqrt{\left(\frac{1}{M} \sum_{m=1}^{M} U_{s h x m}\right)^{2}+\left(\frac{1}{M} \sum_{m=1}^{M} U_{s h y m}\right)^{2}}
\end{gathered}
$$

Next, the harmonic emission level of the consumer can be obtained based on $Z_{s h}$ and $U_{s h}$. According to the superposition principle, the equivalent circuit in Figure 1 can be split into two circuits (Figure 2).

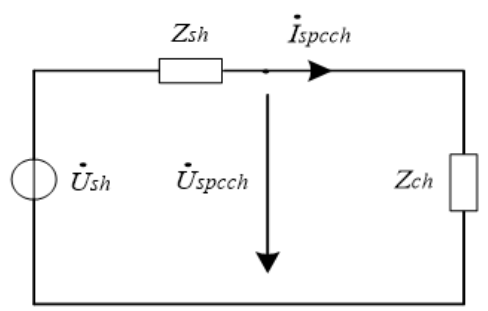

(a) The supply system

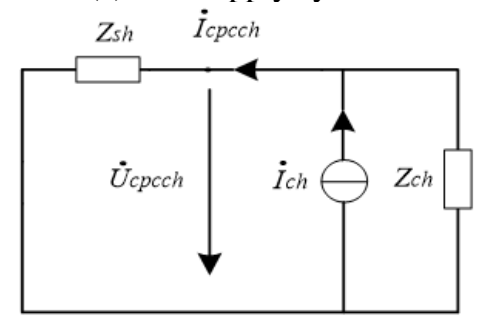

(b) The consumer

Figure 2. The equivalent circuits of the supply system and the consumer
Then, the harmonic emission levels of the supply system and the consumer can be respectively described as:

$$
\begin{gathered}
\dot{U}_{s p c c h}=\frac{Z_{c h}}{Z_{c h}+Z_{s h}} \dot{U}_{s h} \\
\dot{U}_{c p c c h}=\frac{Z_{c h} Z_{s h}}{Z_{c h}+Z_{s h}} \dot{I}_{c h}
\end{gathered}
$$

Equations (12) and (13) can be combined into:

$$
\dot{U}_{p c c h}=\dot{U}_{s p c c h}+\dot{U}_{c p c c h}
$$

The consumer is usually a source of constant harmonic current with a large resistance $Z_{c h}$, while the supply system is often a source of constant harmonic voltage with a small resistance $Z_{s h}$ [16]. Thus, it is obvious that $\left|Z_{c h}\right| \geq\left|Z_{s h}\right|$. Thus, we have:

$$
\begin{gathered}
\left|U_{s p c c h}\right| \approx\left|U_{s h}\right| \\
\left|U_{c p c c h}\right| \approx\left|U_{p c c h}\right|-\left|U_{s h}\right|
\end{gathered}
$$

\section{SIMULATION}

The simulation was carried out on Matlab/Simulink with the circuit in Figure 3. The impedance settings and voltage amplitudes of the supply system for the fundamental and harmonic waves are listed in Tables 1 and 2, respectively. For the supply system, the fundamental frequency and fundamental initial phase were $49.9 \mathrm{~Hz}$ and $10^{\circ}$, respectively. For the consumer, the harmonic current amplitudes were set as follows: $I_{\mathrm{c} 3}=47 \mathrm{~A}, I_{\mathrm{c} 5}=16 \mathrm{~A}, I_{\mathrm{c} 7}=8 \mathrm{~A}, I_{\mathrm{c} 9}=6 \mathrm{~A}$, and $I_{\mathrm{c} 11}=3 \mathrm{~A}$, and the fundamental impedance was set to $2.723+\mathrm{j} 11.345 \Omega$.

For comparison, our method, partial least squares method (PLSM), and improved fluctuation method (IFM) were all applied to estimate the harmonic impedance and the harmonic emission level of the supply system. The estimation results are recorded in Tables 1 and 2. The harmonic emission level of the supply system is listed in the form of relative error (\%)

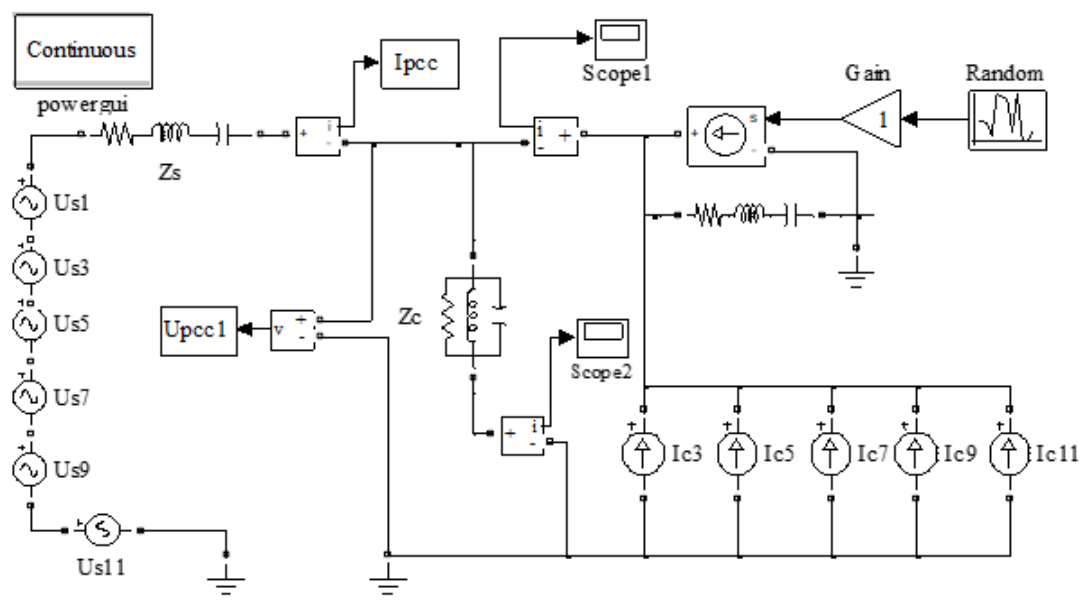

Figure 3. The simulation circuit 
Table 1. Settings and estimates of harmonic impedance of the supply system

\begin{tabular}{ccccc}
\hline Harmonic order & $\begin{array}{c}\text { Set value of } \\
\boldsymbol{Z}_{\mathbf{s h}}(\boldsymbol{\Omega})\end{array}$ & Estimated by the PLSM $(\boldsymbol{\Omega})$ & Estimated by the IFM $(\boldsymbol{\Omega})$ & Estimated by our method $(\boldsymbol{\Omega})$ \\
\hline Fundamental & $0.5+\mathrm{j} 1.371$ & $0.471+\mathrm{j} 1.362$ & $0.481+\mathrm{j} 1.389$ & $0.5+\mathrm{j} 1.371$ \\
3 & $0.5+\mathrm{j} 4.146$ & $0.486+\mathrm{j} 4.012$ & $0.480+\mathrm{j} 4.291$ & $0.497+\mathrm{j} 4.181$ \\
5 & $0.5+\mathrm{j} 6.910$ & $0.5161+\mathrm{j} 6.718$ & $0.530+\mathrm{j} 6.503$ & $0.515+\mathrm{j} 6.610$ \\
7 & $0.5+\mathrm{j} 9.674$ & $0.575+\mathrm{j} 9.970$ & $0.547+\mathrm{j} 9.372$ & $0.519+\mathrm{j} 9.728$ \\
9 & $0.5+\mathrm{j} 12.438$ & $0.468+\mathrm{j} 12.901$ & $0.4772+\mathrm{j} 12.107$ & $0.487+\mathrm{j} 12.173$ \\
11 & $0.5+\mathrm{j} 15.202$ & $0.521+\mathrm{j} 15.937$ & $0.542+\mathrm{j} 16.152$ & $0.531+\mathrm{j} 15.795$ \\
\hline
\end{tabular}

Table 2. Estimated harmonic emission levels of the supply system

\begin{tabular}{ccccc}
\hline Harmonic order & Set value of $\boldsymbol{U}_{\text {sh }}(\mathbf{V})$ & PLSM (\%) & IFM (\%) & Our method (\%) \\
\hline Fundamental & 400 & 0.87 & 1.42 & 1.35 \\
3 & 12 & 1.49 & 1.81 & 1.51 \\
5 & 8 & 1.27 & 1.51 & 0.91 \\
7 & 7 & 2.36 & 2.56 & 1.75 \\
9 & 5 & 2.01 & 4.33 & 1.36 \\
11 & 5 & 3.29 & 3.61 & 1.29 \\
\hline
\end{tabular}

From Tables 1 and 2 , it can be seen that our method estimated the harmonic impedance and harmonic emission level more accurately than the two contrastive methods. For the estimation of harmonic impedance of the supply system, the IMF had the highest error among the three methods; the PLSM had an overall small error, but performed poorly in some cases, such as the real part of the 7 th harmonic impedance. The estimates by our method were close to the set values, except for the 5th harmonic impedance. For the estimation of harmonic emission level of the supply system, our method controlled the relative error within $1.75 \%$, far lower than that of the other methods. Hence, our method can estimate the harmonic emission level of the supply system in an accurate manner.

\section{FIELD TEST}

The field test was carried out in a chemical plant of Yangzhou, China. The plant is powered by $10 \mathrm{kV}$ buses of a $35 \mathrm{kV}$ substation. Under the minimal operation mode, short circuit capacity of the $10 \mathrm{kV}$ outlet is $175.3 \mathrm{MVA}$; under the maximal operation mode, that capacity is $241.6 \mathrm{MVA}$. Two 20MVA power supplies are installed at the PCC. The power distribution network of the plant is shown in Figure 4.

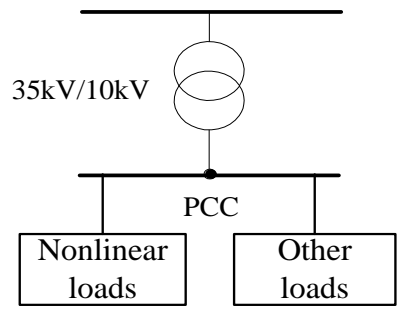

Figure 4. The power distribution network of the plant

The field test was arranged as follows: First, the $10 \mathrm{kV}$ bus voltage of the supply system and the $10 \mathrm{kV}$ outgoing current of the plant were sampled for $5 \mathrm{~s}$ with a measuring device at the interval of $5 \mathrm{~min}$. The harmonics greater than the $20^{\text {th }}$ were filtered out. The sampling frequency was set to $5,000 \mathrm{~Hz}$. Next, the fast Fourier transform (FFT) algorithm with Hanning window [24] was adopted to analyze the measured results by every 500 data points. Then, the harmonic voltage and current at the PCC were analyzed by our method. During the analysis, the harmonic emission level was estimated once for every 500 data points of measured voltage and current.

Due to the limit of space, this paper only lists the measured voltage and current values and the estimated impedance of the supply system corresponding to phase A in the $5^{\text {th }}$ harmonic. The mean voltage, mean current and mean impedance of the supply system for the $5^{\text {th }}$ harmonic wave at the PCC were $105.928 \mathrm{~V}, 36.583 \mathrm{~A}$, and $Z_{s 5}$ is $0.167+\mathrm{j} 2.471 \Omega$, respectively. The real and imaginary parts of the impedance are shown in Figure 5, where the abscissa $n$ is the number of calculations. The $5^{\text {th }}$ harmonic voltage of the system estimated by our method was $10.341 \mathrm{~V}$.

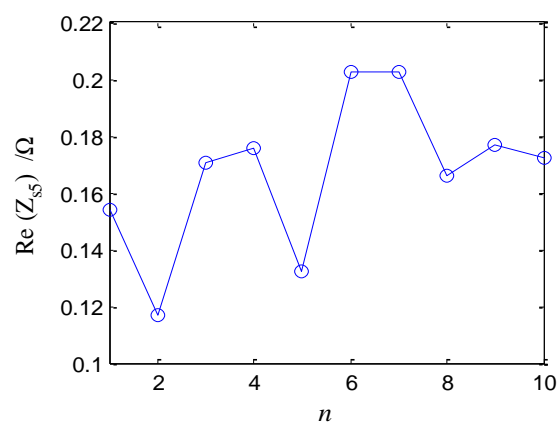

(a) Real part

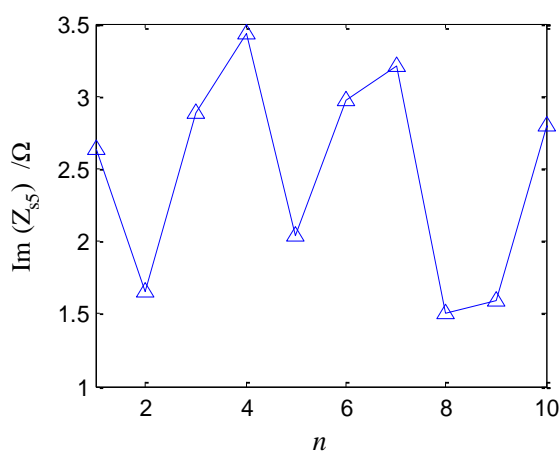

(b) Imaginary part

Figure 5. Estimated $5^{\text {th }}$ harmonic impedance of the supply system

According to the maximal and minimal operation mode capacities of $10 \mathrm{kV}$ bus, the fundamental reference reactance 
of the supply system falls between $0.418 \Omega$ and $0.576 \Omega$. For low harmonics of the supply system, the reactance on the same line satisfies $L_{1}=L_{\mathrm{h}} / h$, where $L_{h}$ is the $h$ th harmonic reactance and $L_{1}$ is the fundamental reactance. The $5^{\text {th }}$ harmonic reactance $L_{\mathrm{s} 5}$ obtained by our method was $2.471 \Omega$. Since $L_{1}=L_{\mathrm{h}} / h, L_{1}$ equals $0.494 \Omega$. The estimated is close to the mean value in the range of $[0.418 \Omega, 0.576 \Omega]$.

According to Equation (16), the harmonic emission level of the consumer can be described as:

$$
U_{c 5}=U_{p c c 5}-U_{s 5}=105.928-10.341=95.587 \mathrm{~V}
$$

Thus, the $5^{\text {th }}$ harmonic emission level of the plant accounts for $90.24 \%$ of the $5^{\text {th }}$ harmonic emission level at the PCC. This means the plant is the main responsible party for harmonic pollution.

In real-world scenarios, the harmonic impedance of the consumer is usually much larger than that of the supply system. Therefore, the harmonic emission level of the consumer can also be calculated by $\left|U_{c h}\right| \approx\left|I_{p c c h}\right|\left|Z_{s h}\right|$. Substituting the values of $Z_{s 5}(2.477 \Omega)$ and $I_{\mathrm{pcc} 5}(36.583 \mathrm{~A})$ into the equation, $U_{c 5}$ can be obtained as $90.616 \mathrm{~V}$, which is close to the estimated value of $95.587 \mathrm{~V}$. Hence, our method was proved to satisfy the engineering practice.

\section{CONCLUSIONS}

This paper puts forward a PCR-based method to estimate the harmonic emission levels of the supply system and the consumer. First, a regression equation was set up based on the complex relationship between harmonic voltage, harmonic current and harmonic impedance of the supply system at the PCC. Then, the PCR was introduced to estimate the harmonic emission levels of both the supply system and the consumer. The proposed method overcomes the multicollinearity between independent variables by removing the principal components with small variance in regression modeling, and retains much of the important information of the original independent variables, which has strong explanatory ability to dependent variables. The accuracy and feasibility of our method were proved through simulation and a field test.

\section{ACKNOWLEDGEMENT}

This work is supported by the key project of State Grid Yangzhou Power Supply Company (Grant No.: J2018112-2).

\section{REFERENCES}

[1] Montoya, F.G., García-Cruz, A., Montoya, M..G., Manzano-Agugliaro, F. (2016). Power quality techniques research worldwide: A review. Renewable and Sustainable Energy Reviews, 54: 846-856. https://doi.org/10.1016/j.rser.2015.10.091

[2] Liu, G.Y., Caldognetto, T., Mattavelli, P., Magnone, P. (2020). Suppression of second-order harmonic current for droop-controlled distributed energy resource converters in DC microgrids. IEEE Transactions on Industrial Electronics, 67(1): 358-368. https://doi.org/10.1109/TIE.2019.2896071

[3] AlyanNezhadi, M.M., Hassanpour, H., Zare, F. (2019).
Grid-impedance estimation in high-frequency range with a single signal injection using time-frequency distribution. IET Science, Measurement and Technology, 13(7): $\quad 1009-1018 . \quad$ https://doi.org/10.1049/ietsmt.2018.5617

[4] Momenpour Akerdi, J., Torabian Esfahani, M., Vahidi, B. (2019). A new method to reduce harmonic magnitude based on simultaneous determination of maximum voltage and current harmonic contribution in interconnected networks. Electric Power Components and Systems, 47(8): 691-703. https://doi.org/10.1080/15325008.2019.1579271

[5] Esparza, M., Segundo, J., Gurrola-Corral, C., VisairoCruz, N., Bárcenas, E., Barocio, E. (2018). Parameter estimation of a grid-connected VSC using the extended harmonic domain. IEEE Transactions on Industrial Electronics, 66(8): 6044-6054. https://doi.org/10.1109/TIE.2018.2870404

[6] Valles, A.P., Revuelta, P.S. (2019). A new distributed measurement index for the identification of harmonic distortion and/or unbalance sources based on the IEEE Std. 1459 framework. Electric Power Systems Resarch, 172: 96-104. https://doi.org/10.1016/j.epsr.2019.03.007

[7] Carta, D., Muscas, C., Pegoraro, P.A., Sulis, S. (2019). Identification and estimation of harmonic sources based on compressive sensing. IEEE Transactions on Instrumentation and Measurement, 68(1): 95-104. https://doi.org/10.1109/TIM.2018.2838738

[8] Meyer, J., Blanco, A.M., Domagk, M., Schegner, P. (2017). Assessment of prevailing harmonic current emission in public low-voltage networks. IEEE Transactions on Power Delivery, 32(2): 962-970. https://doi.org/10.1109/TPWRD.2016.2558187

[9] Moradifar, A., Foroud, A.A., Fouladi, M. (2019). Identification of multiple harmonic sources in power system containing inverter-based distribution generations using empirical mode decomposition. IET Generation Transmission \& Distribution, 13(8): 14011413. https://doi.org/10.1049/iet-gtd.2018.5382

[10] Dirik, H., Duran, I.U., Gezegin, C. (2019). A computation and metering method for harmonic emissions of individual consumers. IEEE Transactions on Instrumentation and Measurement, 68(2): 412-420. https://doi.org/10.1109/TIM.2018.2843538

[11] Enayati, J., Moravej, Z. (2017). Real-time harmonics estimation in power systems using a novel hybrid algorithm. IET Generation Transmission \& Distribution, 11(14): 3532-3538. https://doi.org/10.1049/ietgtd.2017.0044

[12] Nie, X.H. (2019). Detection of grid voltage fundamental and harmonic components using Kalman filter based on dynamic tracking model. IEEE Transactions on Industrial Electronics, 67(2): 1191-1200. https://doi.org/10.1109/TIE.2019.2898626

[13] Cisneros-Magana, R., Medina, A., Anaya-Lara, O. (2017). Time-domain harmonic state estimation of nonlinear load power systems with under-determined condition based on the extended Kalman filter. International Transactions on Electrical Energy Systems, 27(2): 136-143. https://doi.org/10.1002/etep.2242

[14] Cai, L.F., Thornhill, N.F., Kuenzel, S., Pal, B.C. (2017). Real-time detection of power system disturbances based on k-nearest neighbor analysis. IEEE Access, 5: 56315639. https://doi.org/10.1109/ACCESS.2017.2679006 
[15] Moradifar, A., Foroud, A.A., Fouladi, M. (2019). Identification of multiple harmonic sources in power system containing inverter-based distribution generations using empirical mode decomposition. IET Generation Transmission \& Distribution, 13(8): 14011413. https://doi.org/10.1049/iet-gtd.2018.5382

[16] IEC 61000-3-6 Ed.2. (2007). Assessment of harmonic emission limits for the connection of distorting installations to MV, HV and EHV power systems.

[17] Yang, H.G., Pirotte, P., Robert, A. (1996). Harmonics emission levels of industrial loads-statistical assessment. CIGRE, 96: 1936-1996.

[18] Gong, H.L., Xiao, X.Y., Liu, Y.M., Yang, H.G. (2010). A method for assessing customer harmonic emission level based on the dominant fluctuation filtering principle. Proceedings of the CSEE, 30(4): 22-27. https://doi.org/10.13334/j.0258-8013.pcsee.2010.04.002

[19] Zhang, W., Yang, H.G. (2004). A method for assessing harmonic emission level based on binary linear regression. Proceedings of the CSEE, 24(6): 50-53. https://doi.org/10.13334/j.0258-8013.pcsee.2004.06.010

[20] Che, Q., Yang, H.G. (2004). Assessing the harmonic emission level based on robust regression method. Proceedings of the CSEE, 24(4): 39-42. https://doi.org/10.13334/j.0258-8013.pcsee.2004.04.008

[21] Huang, S., Xu, Y.H. (2007). Assessing harmonic impedance and the harmonic emission level based on partial least-squares regression method. In Zhongguo Dianji Gongcheng Xuebao (Proceedings of the Chinese Society of Electrical Engineering), 27(1): 93-97. https://doi.org/10.13334/j.0258-8013.pcsee.2007.01.017

[22] Wold, S., Esbensen, K., Geladi, P. (1987). Principal component analysis. Chemometrics and Intelligent Laboratory $\quad$ Systems, 2(1-3): 37-52. https://doi.org/10.1016/0169-7439(87)80084-9

[23] Johnson, R.A., Wichern, D.W. (2002). Applied multivariate statistical analysis. 5(8): Upper Saddle River, NJ: Prentice hall.

[24] Grandke, T. (1983). Interpolation algorithms for discrete fourier transforms of weighted signals. IEEE Transactions on Instrumentation and Measurement, 32(2): $350-355$. 